\title{
A literature review of MRI techniques used to detect amyloid-beta plaques in Alzheimer's disease patients
}

\author{
Baoting Yu', Yaming Shan ${ }^{2}$, Jun Ding ${ }^{1}$ \\ ${ }^{1}$ Department of Radiology, China-Japan Union Hospital of Jilin University, Changchun, China; ${ }^{2}$ National Engineering Laboratory for AIDS \\ Vaccine, School of Life Sciences, Jilin University, Changchun, China \\ Contributions: (I) Conception and design: J Ding, Y Shan; (II) Administrative support: J Ding; (III) Provision of study materials or patients: B Yu; (IV) \\ Collection and assembly of data: B Yu; (V) Data analysis and interpretation: B Yu, Y Shan; (VI) Manuscript writing: All authors; (VII) Final approval \\ of manuscript: All authors. \\ Correspondence to: Jun Ding, PhD. Department of Radiology, China-Japan Union Hospital of Jilin University, No. 829 of Xinmin Street, Chaoyang \\ District, Changchun, China. Email: dingjun@jlu.edu.cn.
}

\begin{abstract}
Objective: In this review, we aim to provide an overview of the imaging techniques most commonly used to study Alzheimer's disease (AD).

Background Neuroimaging biomarkers can be used to evaluate these abnormalities, improve the ability of early diagnosis and help predict disease progression. These signs mainly include local brain atrophy on structural MRI, hypometabolic foci, and amyloid plaque deposition in the brain detected by specific imaging. These techniques not only have their unique advantages, but can complement each other in multimodal imaging evaluation of patients with cognitive impairment and dementia.

Methods: A literature search was performed on PubMed using the search term combinations "Alzheimer's disease", "Amyloid-beta plaques", and "MRI". We discuss various magnetic resonance imaging (MRI) based techniques, including direct imaging, indirect imaging, amyloid-beta (A $\beta$ ) plaque and radiomics, Hybrid PET/MRI and MRI imaging technology in the future, placing a special emphasis on multimodal imaging, and focus our review on the MRI features of $\mathrm{A} \beta$ plaques (AD biomarkers).

Conclusions: After a lot of research and reasonable selection, multimodal imaging composed of MRI and PET can significantly improve the diagnosis and treatment of AD patients, and the complementary information can be obtained by the new PET/MR instrument at the same time. The findings of this review emphasize that multimodal imaging is likely to be the preferred method for future AD research and clinical practice.
\end{abstract}

Keywords: Alzheimer's disease (AD); amyloid-beta plaques (A $\beta$ plaques); magnetic resonance imaging (MRI); biomarkers

Submitted Apr 07, 2021. Accepted for publication Aug 04, 2021.

doi: 10.21037/apm-21-825

View this article at: https://dx.doi.org/10.21037/apm-21-825

\section{Introduction}

Alzheimer's disease $(\mathrm{AD})$ is a neurodegenerative condition and the main form of dementia. This disease is a serious threat to human health and has become one of the greatest challenges for the healthcare system in the 21st century (1). Clinically, the main symptom of $\mathrm{AD}$ is an initial short-term memory impairment that interferes with activities of daily living. As the disease progresses, other cognitive domains become impaired including language, judgment, orientation, behavior and executive function, ultimately resulting in motor difficulties. Importantly, a preclinical stage characterized by mild cognitive impairment (MCI) lasts for decades before resulting in dementia (2). In this long 
preclinical phase, the typical pathological characteristics are the accumulation of $\mathrm{A} \beta$ plaques and tau tangles that occur throughout the entire process of $\mathrm{AD}$ neurodegeneration. Brain amyloid plaque derived from extracellular deposition of $\beta$-amyloid peptide is considered as one of the most important factors for Alzheimer's disease (AD) onset. However, the true role of $\mathrm{A} \beta$ for $\mathrm{AD}$ development remains controversial. Some scientists hold that the $\mathrm{A} \beta$ is harmful to the organism for it can result in amyloid deposition, nerve fiber tangles, nerve cell death, and eventually the dementia, while others view the $A \beta$ as a helpful factor due to its antibacterial, antioxidant and neurotrophic effects on the brain (3). Both proteinopathies can trigger oxidative stress, microvascular dysfunction, blood-brain barrier (BBB) disruption and the activation of inflammatory response within the brain, ultimately resulting in neuronal damage and consequent neurodegeneration (4).

Based on proteinopathy evidence, the new diagnostic guidelines published in 2011 indicate that biomarker tests should be incorporated in addition to clinical symptom screening, as the definition of $\mathrm{AD}$ has gradually evolved from symptom-based to biology-based (5). Following this development, research into early diagnosis using biomarkers has been undertaken. Biomarkers of functional impairment, neuron loss, and protein deposition can be evaluated by neuroimaging (i.e., MRI and PET) or CSF analysis, and are increasingly used to diagnose Alzheimer's disease in research and professional clinical settings. The cerebrospinal fluid (CSF) biomarkers that can be detected by imaging can provide information about the pathological process in vivo, which is useful in understanding changes in the brain at different stages of the disease (6). In addition, imaging biomarkers are less invasive. Therefore, based on previously developed cognitive, blood and CSF tests and brain imaging methods, a new imaging biomarker method has been developed for $\mathrm{AD}$ diagnosis that is designed to detect $A \beta$ plaque depositions and tau proteins in the living brain (7).

At present, most studies have focused on $\mathrm{A} \beta$ plaque imaging. Although magnetic resonance imaging (MRI) is a method that can be used to detect tau protein-related neurodegeneration, studies of tau proteins are rare, and those that exist have been based on positron emission tomography (PET) rather than MRI (8). Additionally, some studies have shown that tau tangles are not specific biomarkers for $\mathrm{AD}$, and since they are located inside the cells, it is difficult to directly compare them with the production and aggregation of $\mathrm{A} \beta$ plaques (9). Therefore, a literature search was performed on PubMed using the search term combinations "Alzheimer's disease", "Amyloidbeta plaques", and "MRI". this review discusses the existing literature regarding $\mathrm{MRI}$ of $\mathrm{A} \beta$ plaques in $\mathrm{AD}$ patients (Table 1), including direct imaging, indirect imaging, amyloid-beta $(\mathrm{A} \beta)$ plaque and radiomics, Hybrid PET/ MRI and MRI imaging technology in the future, placing a special emphasis on multimodal imaging, and focus our review on the MRI features of $\mathrm{A} \beta$ plaques (AD biomarkers). We present the following article in accordance with the Narrative Review reporting checklist (available at https:// dx.doi.org/10.21037/apm-21-825).

\section{Necessity and feasibility of A $\beta$ plaque MRI}

At present, the imaging technology most commonly used in clinical research to assist in vivo diagnosis is PET (25). However, PET has many disadvantages, such as radiation, low resolution, and high cost, limiting its use as an early screening tool in a conventional clinical setting (26). In contrast, MRI has a higher spatial resolution, is noninvasive, and does not use radiation. Therefore, its use in $\mathrm{A} \beta$ plaque imaging will be an important step towards early diagnosis and improved prognosis for $\mathrm{AD}$. $\mathrm{A} \beta$ is produced by the metabolism of amyloid precursor protein (APP). App can be cleaved by extracellular protease of $\alpha$-secretase to produce soluble extracellular fragment (Sapp $\alpha$ ), which is cleaved by $\beta$-secretase (BACE1) to produce soluble extracellular fragment (Sapp+) and cell membrane binding fragment (C99). The cell membrane binding fragment is cleaved by $\gamma$-secretase to release the amyloid intracellular domain and $\mathrm{A} \beta, \mathrm{A} \beta$ aggregates to form oligomers, fibrils, and plaques. In $\mathrm{AD}$, the changes of $\mathrm{A} \beta$ concentration appeared in cerebrospinal fluid (CSF), followed by the accumulation of brain $\mathrm{A} \beta$, the increase of CSF, the decrease of hippocampus and gray matter volume, the decrease of glucose metabolism, memory impairment, and dementia. Several studies have confirmed that these biomarkers can indeed be used to reliably measure disease-related brain changes in vivo $(27,28)$.

MR imaging of $\mathrm{A} \beta$ plaques was performed using $\mathrm{T} 2$ * contrast medium technique. So far, the non-invasive detection ability of $A \beta$ plaques in MR images is mainly attributed to the focal iron deposition accompanied with plaques. The $\mathrm{T} 2 *$ shortening effect of paramagnetic iron is the main source of the contrast between plaques and surrounding tissues. Amyloid plaque itself does not cause a susceptibility effect. The aggregation of $\beta$-amyloid protein 
Table 1 A summary of the different sequences methods for A $\beta$ MRI analysis in AD for 10 years

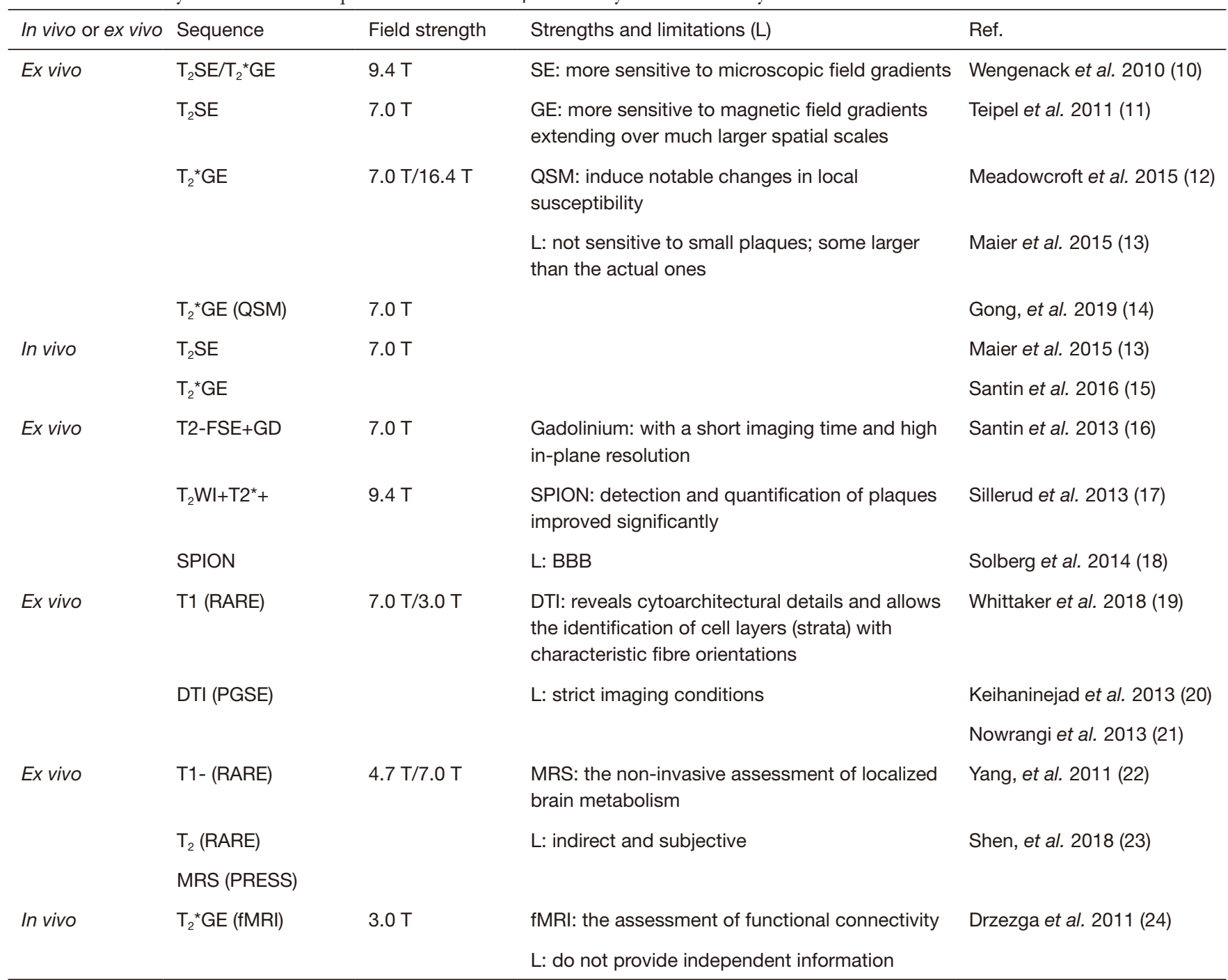

MRI, magnetic resonance imaging; AD, Alzheimer's disease; SE, spin-echo; GE, gradient echo; QSM, quantitative sensitivity mapping; FSE, fast SE; GD, gadolinium; SPION, superparamagnetic iron oxide nanoparticle; DTI, diffusion tensor imaging; MRS, magnetic resonance spectroscopy; fMRI, functional MRI; RARE, rapid acquisition relaxation enhancement; PGSE, pulsed gradient spin echo; PRESS, point resolved spectroscopy; L, limitations.

can increase the electron density and cause a significant change in the local sensitivity value, which is large enough to produce the contrast relative to the surrounding normal tissue, and can be displayed by quantitative sensitivity mapping (QSM) MR imaging. Single A $\beta$ plaques can also be displayed in high-resolution sensitivity maps. The diamagnetism of $\mathrm{A} \beta$ protein can also be observed in brain samples of $\mathrm{AD}$ patients.

In recent years, MRI has proved to be effective in the detection of early $\mathrm{AD}$-related brain changes that are used in the diagnosis, prediction, and determination of the disease stage. A $\beta$ plaque imaging can accurately detect early pathological changes in $\mathrm{AD}$ patients by observing the distribution and density of plaques in the brain, determining the stage of the disease and predicting its progress. In addition, $A \beta$ plaque imaging can be used for specific clinical trials and has a high predictive value and level of proficiency in diagnosing $\mathrm{AD}$ and other types of dementia (29). Moreover, a large meta-analysis showed that $\mathrm{A} \beta$ plaque deposition in the brain is closely related to age and that the correlation curve of $A \beta$ plaque incidence with age is very similar to that of dementia incidence with age 
$(30,31)$. However, plaque deposition begins at least 20 years earlier than dementia (32).

Individual plaques in transgenic mice with $\mathrm{AD}$ can be gradually visualized using high-field MRI systems both in vivo and ex vivo (33). Histological measurements have shown that plaques of 20 and $35 \mu \mathrm{m}$ in diameter are detectable using ex vivo and in vivo MRI, respectively (34). However, the imaging of these amyloid plaques is limited by the MRI resolution in murine studies. Moreover, detection requires careful optimization of imaging protocols and analysis. The increase in paramagnetism caused by the distribution of iron in and around the plaques seems to be the dominant contributing factor (35). The reduction of artifacts and blurring caused by breathing and heartbeat also affects $A \beta$ plaque imaging in vivo (36). In this case, the optimization of MRI scanning sequences depends on electrocardiogram or respiratory gating techniques. Figure 1 provides a summary of the principles and applications of $A \beta$ plaque MRI as well as those of new imaging methods expected to be further explored and effectively used in $\mathrm{AD}$ clinical research.

\section{Direct imaging of Aß plaques}

\section{Relaxation time}

To find subtle changes in tissue characteristics, MRI has primarily been used to study changes in relaxation time. The goal of mapping the relaxation time in neurodegenerative diseases is usually to detect changes in tissue characteristics, particularly with regard to determining whether the presence of abnormal protein depositions in tissues can be identified by monitoring changes in relaxation time. The use of transgenic animal models of AD allows in vivo imaging to directly characterise neuropathology and provides an effective experimental platform for testing hypotheses relating to pathophysiology. The T1 and T2 relaxation times vary with the different depths of human and mouse brains. This reflects the layered structure of the cortex, and changes in these relaxation rates are related to changes in the myelin sheath and nerve cell density. Many studies using different transgenic animal models have shown that an increase in the number of $\mathrm{A} \beta$ plaques corresponds to changes in the $\mathrm{T} 1$ and $\mathrm{T} 2$ relaxation times (33).

Detailed histological and pathological analyses of human tissues have shown that a decrease in $\mathrm{T} 2$ relaxation time in $\mathrm{AD}$-affected brain tissue is related to an increase in iron (37), and that the accumulation of iron in activated microglia and the myelin sheath of the middle cortex is related to the Braak stage (38). Ferric iron $\left(\mathrm{Fe}^{3+}\right)$ can change the microscopic magnetic field homogeneities as a ferromagnetic ion, resulting in the dephasing of the signal of water protons in the vicinity, which leads to the T2 relaxation time shortening $(39,40)$. Dhenain et al. $(41)$ and Faber et al. (42) successfully detected plaques in thalamus of transgenic mice using gradient echo and fast low resolution gradient echo, respectively, which were large and high in $\mathrm{Fe}$ content. Iron can be stored in amyloid polymers and can increase the toxicity of $A \beta$ plaques (43). Recently, some studies have found that there are $\mathrm{Fe}^{3+}, \mathrm{Fe}^{2+}$ and ferritin in amyloids. Therefore, there has been speculation that focal iron deposition in the plaques could lead to the transverse relaxation changes associated with $\mathrm{A} \beta$ plaques (44). The data show that iron content is not the only cause of the hypointensity seen in the image, as the dense fibrillar nature of the $A \beta$ plaques also has an influence on transverse relaxation times in $\mathrm{AD}$-affected tissue (12).

Benveniste et al. (45) used MRI for the first time to display $A \beta$ plaques in the human brain in vitro. Subsequently, several research teams found that single amyloid plaques in $\mathrm{AD}$ transgenic mice could be distinguished in $\mathrm{T} 2$ and T2* weighted MRI without the use of exogenous contrast agents by improving the magnetic field intensity, resolution, and acquisition times (46). Plaques have been visualized ex vivo using T2-weighted spin-echo (SE), fast SE (FSE), and gradient-echo (GRE) techniques. There are many techniques for plaque detection in vivo, including T1 weighting, macroscopic T2 mapping, FSE imaging, SE imaging, and GRE imaging.

\section{Susceptibility mapping}

In recent years, a method of quantifying the local magnetic susceptibility values from MR phase images, called quantitative susceptibility mapping (QSM), has led to plaque visualization as $\mathrm{A} \beta$ plaques and iron sediments can affect the magnetic susceptibility of tissues (47-49). These values can be used to non-invasively evaluate the ability of $\mathrm{A} \beta$ plaques to aggregate, aiding the diagnosis of $\mathrm{AD}$ (14). Another diagnostic technique, susceptibility-weighted imaging (SWI), is used to increase image contrast by the displacement of voxels caused by the naturally existing iron in the plaques (20), exploiting the susceptibility differences between tissues. SWI may be more suitable for in vivo human imaging than in vivo mouse imaging due to the increased distance from the air/tissue interface to the region of interest (ROI) (50). An increasing number of studies show that magnetic transfer imaging (MTI) has been successfully used to characterise neurodegenerative diseases, 


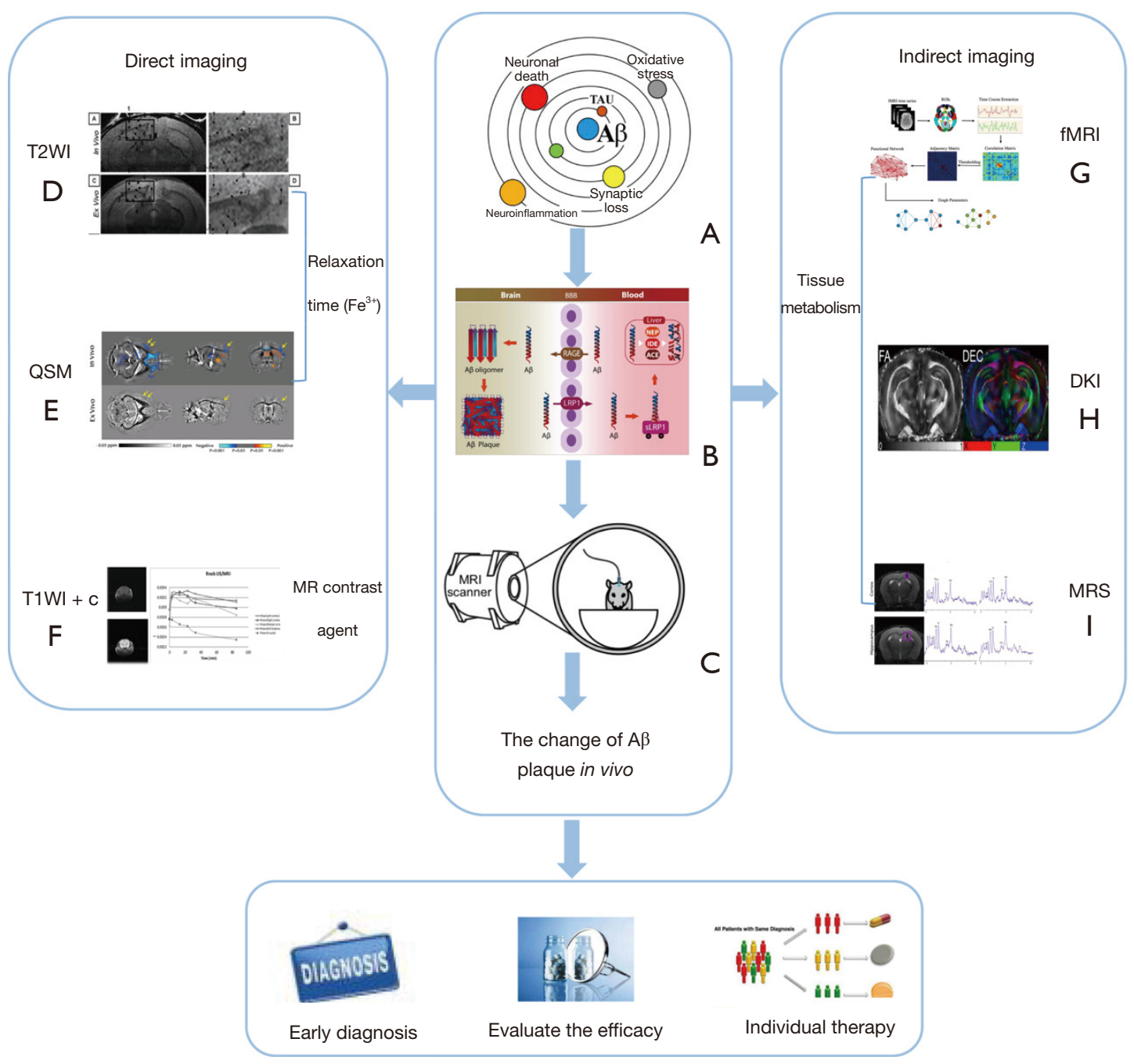

Figure 1 The flow chart of the Magnetic resonance (MR) imaging of A $\beta$ plaque in AD transgenic mice. (A) The amyloidocentric theory of Alzheimer's disease (AD) (18). The amyloid cascade hypothesis represented as the geocentric Ptolemy's theory of the solar system, which placed the Earth at the center and was accepted for many centuries. (B) The distribution and degradation pathway of A $\beta$ peptide monomer between brain and blood circulation (8). (C) MRI scan pattern of mice. (D) 24-month-old AD mouse. In vivo MRI (A,B), ex vivo MRI (C,D) images (20). (E) Comparison between in vivo and ex vivo susceptibility maps (14). (F) In vivo MRI scanning up to 80 min after Fused in sarcoma (FUS) activation (13). (G) Procedure to obtain the functional network and graph theory parameters (15). (H) Quantitative MRI maps of the middle slice of a representative Tg2576 mouse brain. Maps: fractional anisotropy (FA), directionally encoded color (DEC) (16). (I) Localization and representative magnetic resonance spectroscopy (MRS) spectra from the hippocampus and frontal cortex (red box) of T2weighted scans of Amyloid precursor protein/presenilin 1(APP/PS1) and wild-type (WT) mice (17).

including $\mathrm{AD}(50)$.

\section{MRI contrast agents}

At present, individual $\mathrm{A} \beta$ plaques in $\mathrm{AD}$ transgenic mice can be directly imaged by MRI with a good anatomical correlation between the location and size of the plaques (46). However, due to the relatively small size of some plaques, increasing the contrast between the plaques and the surrounding brain tissue, or enhancing the contrast between the sizes of different structures, is needed to measure individual plaques in human brains. Targeted MRI contrast agents can improve the specificity of $\mathrm{A} \beta$ plaques. As a targeted contrast agent, it should have the following characteristics: it has specific binding site with $A \beta$ plaque 
and has high affinity; it has less non-specific binding and can be cleared quickly; it has certain fat solubility and can effectively pass through the blood-brain barrier; it is non-toxic and has enough signal contrast intensity for quantitative analysis. Ionic gadolinium chelates (13) and superparamagnetic iron oxide nanoparticles (SPIONs) (15) are the main MRI contrast agents used in the imaging of $\mathrm{A} \beta$ plaques in vivo and ex vivo to date. Several other MRI contrast agents have reportedly been used, e.g., putrescineGd-A $\beta$, Gd-DTPA-A $\beta$ and MION-A $\beta$.

There is no rapid and effective method to evaluate the ability of contrast agents to pass through the BBB, and there is a lack of extensive animal experiments and toxicity studies in vivo. The role of contrast agents in the brain largely depends on the interaction with the $\mathrm{BBB}$. If $\mathrm{A} \beta$ plaque aggregation is targeted using binding components that are highly specific, such as small proteins, the reagents containing protein or peptide sequences may be blocked by the BBB. Therefore, an important step in the development of imaging biomarker is the introduction of a contrast agent that targets $A \beta$ plaques efficiently and accurately by penetrating the BBB. This would not only reduce the influence of small plaques and iron content but also would more directly reflect the actual condition of the plaques.

In conclusion, direct imaging techniques are key to the study of $\mathrm{AD}$ biomarkers, and improving the direct imaging of $\mathrm{A} \beta$ plaques is particularly important for understanding the mechanism of plaque formation and the changes associated with disease progression.

\section{Indirect imaging of A plaques}

\section{Imaging of neural network connections: functional MRI}

Functional MRI (fMRI) is a method of indirectly measuring brain nerve activity by measuring changes in blood oxygenation levels (blood-oxygenation-level-dependent imaging, or BOLD, is the most common method), mainly to observe the signal changes resulting from blood oxygenation levels (18). The BOLD signal reflects dynamic changes in blood oxygenation levels caused by neuronal and synaptic activities and has been widely used in clinical practice. The BOLD contrast mechanism has a complex relationship with functional brain activity, oxygen metabolism, and neurovascular factors. Accurate interpretation of the BOLD signal for neuroscience and clinical applications necessitates a clear understanding of the sources of BOLD contrast and its relationship to underlying physiology (51). In recent years, with the increased use of fMRI, brain connectivity impairment in patients with $\mathrm{AD}$ is obvious and gradually the potential white matter networks are accompanied by disease progression. A large number of existing MRI studies have shown that to a large extent, this change enables in vivo observation of disease progression (52). Quantifying the strength of the functional connections between regions of the neural network is useful when studying $\mathrm{AD}$, because a decrease in the strength of the functional connections may reflect a decrease in the integrity of the neural network (53).

Looking at several groups of studies, the combined analysis of $\mathrm{fMRI}$ and amyloid studies showed that amyloid deposition in the brain was related to brain connectivity damage. Another study of an asymptomatic population with complete cognitive function but high levels of amyloid deposition in the brain found that the default mode network (DMN) connection was disrupted (24). These studies suggest that there is a correlation between amyloid deposition and DMN connectivity and that these changes occur prior to the onset of cognitive impairment.

The expression of the $\varepsilon 4$ allele of the apolipoprotein $\mathrm{E}$ (APOE $\varepsilon 4$ ) is a hereditary indicator of $\mathrm{AD}$. As a pathological partner of $A \beta$ plaques, APOE $\varepsilon 4$ can not only significantly promote the deposition of $\mathrm{A} \beta$ plaques but also reduce the rate at which they clear (54). Recent studies have shown that the decrease in DMN connectivity in individuals carrying $\mathrm{APOE} \varepsilon 4$ is related to an increase in amyloid protein levels in the cortex (4). fMRI imaging indirectly assesses plaque deposition and changes in the brain by monitoring the $\mathrm{DMN}$ connection status and is therefore potentially useful in the detection of $\mathrm{AD}$.

However, fMRI is dependent on hemodynamics and oxygen metabolism. The contrast enhancement produced by fMRI imaging depends on cerebral blood flow, cerebral blood volume and cerebral metabolic level/oxygen consumption. These fMRI are indirect manifestations, which cannot directly reflect the activity of neurons. In addition, the results of different studies on fMRI vary greatly, and the use of $\mathrm{FMRI}$ in the early diagnosis of $\mathrm{AD}$ needs more research to further verify.

\section{Imaging of water molecule movement: DWI, DTI and DKI}

Diffusion-weighted imaging (DWI) is a method that uses MRI to observe and quantitatively analyse the micromotion of water molecules in living tissues, mainly Brownian motion or water diffusion. Among them, diffusion tensor imaging (DTI) is the most widely used in a clinical setting, 
as it can display a wide variety of microstructures. This is essential for identifying the fibre tracks of white matter (WM) and disruption due to disease. The mean diffusivity (MD) and fractional anisotropy (FA) reflect changes in the structure of WM. An increase in MD and a decrease in FA indicate degeneration in the structure of WM (55). A large number of studies have shown that AD affects a specific part of the neural network (56). Mathematical modelling supports the hypothesis that AD-related pathological substances, such as amyloid proteins, propagate protein misfolding along structural connections and fragile networks in the brain (57). Several studies have found that there are differences between MCI groups and healthy groups following DTI evaluation of brain biomarkers in $\mathrm{AD}$ patients, and MD is more sensitive than FA when discriminating between the two groups (58).

So far, in order to improve diagnostic accuracy and specificity when assessing changes in WM in AD patients, a variety of DTI methods have been used (19). However, some studies have shown that DTI sequences do not detect significant changes in younger age groups because age-dependent $\mathrm{A} \beta$ plaque accumulation is related to $\mathrm{WM}$ abnormalities (10). It is difficult for DTI alone to accurately reflect the actual state of plaques when age, gender and other relevant factors are taken into consideration. In addition, the clinical application of DTI parameters is limited. First, DTI is a single exponential diffusion imaging method based on the Gaussian model. It is difficult to accurately express the structural characteristics of tissues with only simple MD and FA because there is non-Gaussian distribution of water diffusion in tissues (11). Second, DTI measurements lack biological specificity. For example, an increase in MD and a decrease in FA might not only indicate degeneration in the structure of WM, but may also be related to neuronal injury or demyelination (12).

Diffusion kurtosis imaging (DKI) does not have the problems associated with DTI and takes the measurements of non-Gaussian distribution of water diffusion in tissues into account. DKI has the advantage that it is more sensitive in detecting neuropathy, but it has a poor parameter specificity and a longer acquisition time.

\section{Imaging of tissue metabolism: ${ }^{1} \mathrm{H}-\mathrm{MRS}$ and CEST}

Proton magnetic resonance spectroscopy $\left({ }^{1} \mathrm{H}-\mathrm{MRS}\right)$ imaging uses neurochemical changes in vivo and chemical shifts to quantitatively analyse specific nuclei and compounds. By observing the concentrations of different compounds in the nucleus and assessing the slight differences, this method can non-invasively measure the metabolic changes in compounds including $\mathrm{N}$-acetyl aspartate (NAA), choline (Cho), creatine (Cr) and lactate in brain tissue in vivo (59). This non-invasive technique has become the standard in $\mathrm{AD}$-related metabolic research methods. In $\mathrm{AD}$ patients, the main clinical finding from ${ }^{1} \mathrm{H}-\mathrm{MRS}$ is a decrease in NAA. Other metabolites such as myo-inositol (mI), Cho, glutamate $(\mathrm{Glu})$ and $\mathrm{Cr}$ also change in patients with MCI and $\mathrm{AD}(60)$.

According to the results from animal experiments, changes to the metabolic spectrum are related to the physiological and pathological processes of AD (61). Research comparing the hippocampi of $\mathrm{AD}$ mice to those of wild-type mice (62) showed that the NAA/Cr and $\mathrm{Glu} / \mathrm{Cr}$ ratios decreased significantly, while the $\mathrm{mI} / \mathrm{Cr}$ ratio significantly increased. However, these significant differences only occurred in the brain cortices of the AD mice. This may be due to earlier occurrences of neurometabolic disorders in the hippocampus during AD progression (23). The above results also show that the metabolic changes appeared earlier than the clinical manifestations of dementia. Therefore, ${ }^{1} \mathrm{H}-\mathrm{MRS}$ is a valuable tool in the early clinical evaluation of $\mathrm{AD}$ (Figure 2).

Chemical exchange saturation transfer (CEST) is a novel MR technique that has the potential to provide molecular information for the diagnosis of AD (63). CEST uses radiofrequency pulse to magnetically label exchangeable protons to detect molecular information. CEST has the advantages of high cost-effectiveness, non-invasive and high sensitivity, which actually overcomes some concentration limits of $1 \mathrm{H}-\mathrm{MRS}$, and has great potential and prospect in $\mathrm{AD}$ diagnosis (64).

\section{$\mathrm{A} \beta$ plaques and radiomics}

With the development of artificial intelligence and deep learning, modern analysis methods such as machine learning and data mining have gradually been incorporated into medical use. Studies have shown that MRI can be used to observe the differences in other markers between a control group and patients with $\mathrm{AD}$ and different types of dementia (65). For example, AD can be distinguished from Lewy body dementia and vascular cognitive impairment by observing the degree of atrophy in the medial temporal lobe with high sensitivity and specificity (>90\%) (66). A combination of resting fMRI, neural aging and deep learning methods can detect prognostic indicators and risk factors, enabling the early diagnosis of neurodegenerative 

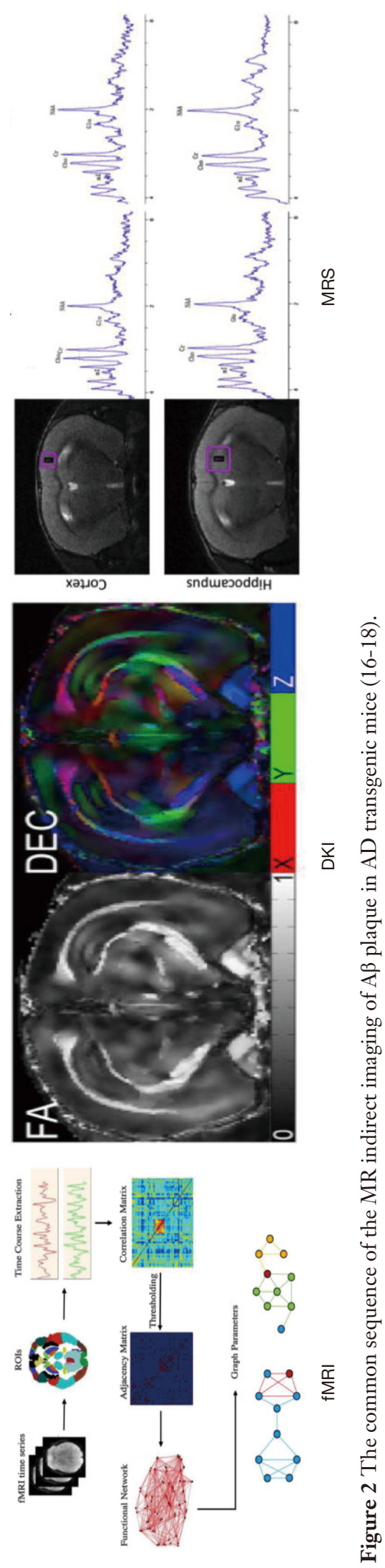

changes and assisting with treatment decisions (67). Using these methods to monitor the progression of $\mathrm{AD}$ and the effect of targeted therapies may also assist with the development of more effective treatments. It is believed that the combination of imaging biomarker, artificial intelligence and deep learning algorithms will provide a more complete map of structural and metabolic changes in the near future. This will improve the ability to combine images to derive information, taking into consideration the impact of genetic, gender and lifestyle factors (Figure 3).

At present, the main clinical treatment methods include cholinesterase inhibitors (CIS), which can be used throughout the course of the disease, and memantine (MEM), which is suitable for patients with moderate to severe $\mathrm{AD}$. The main purpose of these medications is to improve the quality of life and prognosis of patients, but they have little impact on the disease process (68). However, $\mathrm{A} \beta$ aggregation inhibitors, such as polypeptides and antibodies, can be used to treat $\mathrm{AD}$ at an earlier stage (69). Polypeptides have more clinical possibilities, participate more in the synthesis and design of compounds, are more highly targeted, have more biological effects, fewer side effects and are more practical (70). For example, studies show that combined treatment with monoclonal antibodies and BACE1 inhibitors can significantly reduce the production of amyloid proteins in mice (71). The authors believe that if a variety of monoclonal antibodies are used to target $\mathrm{A} \beta$ plaques and promote plaque decomposition, this 'cocktail therapy' will probably play an important role in the treatment of AD. If so, MRI technology will be indispensable in monitoring the therapeutic effects of amyloid removal in the brain, guiding clinical adjustments to the treatment plan.

\section{Multimodal imaging}

MRI of $A \beta$ plaques presents many limitations in vivo at present. For example, its success is related to plaque size and MRI resolution; some small plaques cannot be imaged and some plaques are slightly too large in volume. Moreover, many factors influence in vivo imaging and some artifacts and interferences cannot be avoided. In light of these problems, a multimodal imaging approach would combine complementary methods to continuously verify MRI results, optimise sequences and explore more efficient and accurate ways to detect plaques. This would include the use of contrast agents and MRI technology.

At present, short-term tests specifically targeting $\mathrm{AD}$ 


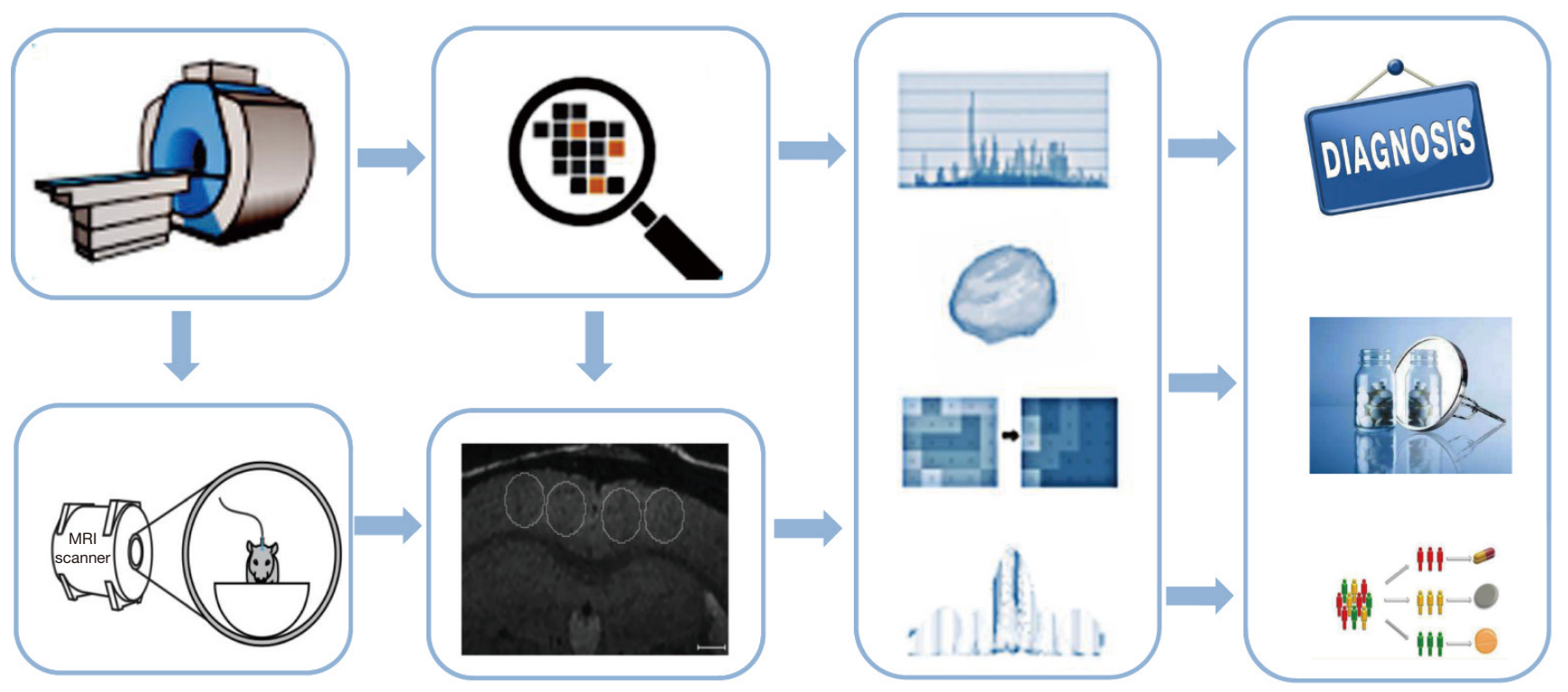

Figure 3 The combination of MR imaging of $A \beta$ plaque and radiomics (20).

have been conducted with small sample sizes, using $A \beta$ plaque biomarkers to confirm the therapeutic targets and effects (72). Currently, MR images (in vivo and ex vivo) and histological sections have still not been unequivocally matched to the display of plaques, and there are still some plaques that can only be seen histologically. Therefore, the multimodal combination of histology and MR images would eliminate the shortcomings of each method. MRI can facilitate clinical non-invasive measurement of $\mathrm{AD}$-related neuropathology by showing changes in plaques and their distribution. However, establishing imaging biomarkers needs further work.

\section{Hybrid PET/MRI}

PET imaging is a mature technique to detect cerebral amyloid beta (a $\beta$ ) plaques in the body. Some preclinical and postmortem data showed that redox active iron accumulated near a $\beta$ plaque. Quantitative susceptibility mapping (QSM) at high-field MRI enables iron deposits to be depicted with high spatial resolution.

Mtpet/MRI hybrid imaging can achieve high spatial resolution quantitative imaging of amyloid plaques in Alzheimer's disease mouse model. Hybrid PET and MRI techniques allow true simultaneous pet and MRI and have become an attractive way to correlate tracer uptake and anatomy. Its clinical availability provides direct benefits for translational research. Combined with PET/MR technology, pet radiotracer has high sensitivity, wide target area, high contrast of MRI soft tissue and strong ability of functional imaging. Real PET/MRI synchronization opens the door to synchronous dynamic research of pet and MRI, allowing complementary dynamic parameters to be obtained from pet and MRI neurology research. For example, in the earlier PET/CT hybrid technology (PET$\mathrm{CT}$ ), pet and CT imaging were performed in sequence, so they were offset in time and space. On the contrary, the real synchronization of pet and MRI can be achieved by these new hybrid systems, eliminating the mismatch effect of fine motion between two scans. This mismatch confuses the correlation between tracer uptake and anatomy, especially at the spatial scale of the mouse brain and its regions. Of course, MRI provides fine contrast between soft tissues, such as different areas of the brain-details that cannot be obtained with CT. Therefore, PET-MRI provides anatomical imaging with high spatial resolution and high contrast, which complements the absolute quantitative tracer uptake obtained by PET. Therefore, PET-MRI is very valuable for the study of $\mathrm{AD}$ mouse model, because it can quantify the tracer uptake in different brain regions.

The latest technology is PET/MR integrated machine, but its clinical application is still limited. This all-in-one machine can obtain MRI and PET data simultaneously in the traditional MRI scanning time, increasing the diagnostic information without additional acquisition and detection time. Because the acquired images are directly 
registered, standard MRI and PET images can be acquired simultaneously in one system, which can increase a lot of diagnostic information. PET/MR has a unique ability to simultaneously evaluate the dynamic process of brain, especially with the use of new tracer for dynamic PET acquisition and functional MRI. These new technologies have obvious advantages in the application of scientific research, but at the same time, we need to continue to study whether the information has clinical application value.

\section{Summary and prospects}

Although the pathogenesis of $\mathrm{AD}$ has not yet been fully understood, the amyloid cascade hypothesis is now dominant (73) and is the fundamental focus of neurodegenerative studies. Dementia and neurodegenerative diseases are not easy to detect in the early stages, and diagnosis can be difficult, especially when the symptoms are atypical. With the understanding of $\mathrm{AD}$ deepening, diagnostic criteria are moving towards the biological characteristics. Therefore, using MRI to diagnose amyloid plaques could have wider clinical implications.

At present, the differential diagnosis of AD is mainly based on multimodal methods, including pathological manifestations, biochemical indicators and imaging markers. Collectively considering MRI analysis of $\mathrm{A} \beta$ amyloid plaques, patient age, iron deposition in the brain, genetic mutations and other related factors will contribute to constructing an $\mathrm{AD}$ histology model designed to establish a multimodal diagnosis structure. This could greatly improve the early diagnosis of $\mathrm{AD}$ and enable the development of individualised treatments. In the near future, combining imaging biomarker with artificial intelligence or deep learning algorithms will provide a more complete map of structural and metabolic changes, enhancing our ability to integrate image data, derive additional information, and fully consider the impact of genetic, gender and lifestyle factors (Figure 3).

But there are still some problems to be solved. (I) So far, MRI research has not been used in the study of living human brain. The reason is that the scanning time of the existing technology is too long (the most appropriate time should be limited to 10 minutes), which is the biggest obstacle in the imaging of human brain plaque in vivo; How to further improve the sensitivity of high field MR microscopy to a $\beta$ plaque and the effect of high field intensity on human body need to be further studied. (II)
Whether MRI targeted nano contrast agent is safe and effective in human body, and the toxicity of ligands in vivo need to be further studied.

This review has summarised the principles and characteristics of MRI analysis techniques for visualising $A \beta$ plaques, which provide more possibilities for the optimisation of MRI technology and treatment of AD. In conclusion, MR imaging can not only reveal the size and distribution of plaques but also show any changes in vivo. In addition, MRI is relatively low in cost and very useful clinically. The authors believe that plaque imaging will become the preferred method for $\mathrm{AD}$ diagnosis and followup in the near future.

\section{Acknowledgments}

This manuscript has been proofread and edited by a professional English editing company from Proofed Inc.

Funding: Health and Health Technology Innovation Project of Jilin Province (2018J078); Discipline Layout Project of Natural Science Foundation of Technology Department of Jilin Province (20200201373JC).

\section{Footnote}

Reporting Checklist: The authors have completed the Narrative Review reporting checklist. Available at https:// dx.doi.org/10.21037/apm-21-825

Conflicts of Interest: All authors have completed the ICMJE uniform disclosure form (available at https://dx.doi. org/10.21037/apm-21-825). The authors have no conflicts of interest to declare.

Ethical Statement: The authors are accountable for all aspects of the work in ensuring that questions related to the accuracy or integrity of any part of the work are appropriately investigated and resolved.

Open Access Statement: This is an Open Access article distributed in accordance with the Creative Commons Attribution-NonCommercial-NoDerivs 4.0 International License (CC BY-NC-ND 4.0), which permits the noncommercial replication and distribution of the article with the strict proviso that no changes or edits are made and the original work is properly cited (including links to both the formal publication through the relevant DOI and the license). 
See: https://creativecommons.org/licenses/by-nc-nd/4.0/.

\section{References}

1. Femminella GD, Thayanandan T, Calsolaro V, et al. Imaging and Molecular Mechanisms of Alzheimer's Disease: A Review. Int J Mol Sci 2018;19:3702.

2. Scheltens P, Blennow K, Breteler MM, et al. Alzheimer's disease. Lancet 2016;388:505-17.

3. Small SA, Duff K. Linking Abeta and tau in late-onset Alzheimer's disease: a dual pathway hypothesis. Neuron 2008;60:534-42.

4. Jokar S, Khazaei S, Behnammanesh H, et al. Recent advances in the design and applications of amyloid- $\beta$ peptide aggregation inhibitors for Alzheimer's disease therapy. Biophys Rev 2019. [Epub ahead of print]. doi: 10.1007/s12551-019-00606-2.

5. McKhann GM, Knopman DS, Chertkow H, et al. The diagnosis of dementia due to Alzheimer's disease: recommendations from the National Institute on AgingAlzheimer's Association workgroups on diagnostic guidelines for Alzheimer's disease. Alzheimers Dement 2011;7:263-9.

6. Frisoni GB, Boccardi M, Barkhof F, et al. Strategic roadmap for an early diagnosis of Alzheimer's disease based on biomarkers. Lancet Neurol 2017;16:661-76.

7. Clark CM, Pontecorvo MJ, Beach TG, et al. Cerebral PET with florbetapir compared with neuropathology at autopsy for detection of neuritic amyloid- $\beta$ plaques: a prospective cohort study. Lancet Neurol 2012;11:669-78.

8. Gobbi LC, Knust H, Körner M, et al. Identification of Three Novel Radiotracers for Imaging Aggregated Tau in Alzheimer's Disease with Positron Emission Tomography. J Med Chem 2017;60:7350-70.

9. Larbanoix L, Burtea C, Ansciaux E, et al. Design and evaluation of a 6-mer amyloid-beta protein derived phage display library for molecular targeting of amyloid plaques in Alzheimer's disease: Comparison with two cyclic heptapeptides derived from a randomized phage display library. Peptides 2011;32:1232-43.

10. Wengenack TM, Reyes DA, Curran GL, et al. Regional differences in MRI detection of amyloid plaques in $\mathrm{AD}$ transgenic mouse brain. Neuroimage 2011;54:113-22.

11. Teipel SJ, Kaza E, Hadlich S, et al. Automated detection of amyloid- $\beta$-related cortical and subcortical signal changes in a transgenic model of Alzheimer's disease using highfield MRI. J Alzheimers Dis 2011;23:221-37.

12. Meadowcroft MD, Peters DG, Dewal RP, et al. The effect of iron in MRI and transverse relaxation of amyloid-beta plaques in Alzheimer's disease. NMR Biomed 2015;28:297-305.

13. Maier FC, Keller MD, Bukala D, et al. Quantification of $\beta$-Amyloidosis and rCBF with Dedicated PET, 7 T MR Imaging, and High-Resolution Microscopic MR Imaging at 16.4 T in APP23 Mice. J Nucl Med 2015;56:1593-9.

14. Gong NJ, Dibb R, Bulk M, et al. Imaging beta amyloid aggregation and iron accumulation in Alzheimer's disease using quantitative susceptibility mapping MRI. Neuroimage 2019;191:176-85.

15. Santin MD, Vandenberghe ME, Herard AS, et al. In vivo Detection of Amyloid Plaques by Gadolinium-Stained MRI Can Be Used to Demonstrate the Efficacy of an Antiamyloid Immunotherapy. Front Aging Neurosci 2016;8:55.

16. Santin MD, Debeir T, Bridal SL, et al. Fast in vivo imaging of amyloid plaques using $\mu$-MRI Gd-staining combined with ultrasound-induced blood-brain barrier opening. Neuroimage 2013;79:288-94.

17. Sillerud LO, Solberg NO, Chamberlain R, et al. SPIONenhanced magnetic resonance imaging of Alzheimer's disease plaques in A $\beta P P / P S-1$ transgenic mouse brain. J Alzheimers Dis 2013;34:349-65.

18. Solberg NO, Chamberlin R, Vigil JR, et al. Optical and SPION-enhanced MR imaging shows that trans-stilbene inhibitors of NF- $\mathrm{kB}$ concomitantly lower Alzheimer's disease plaque formation and microglial activation in A $\beta P P / P S-1$ transgenic mouse brain. J Alzheimers Dis 2014;40:191-212.

19. Whittaker HT, Zhu S, Di Curzio DL, et al. T1, diffusion tensor, and quantitative magnetization transfer imaging of the hippocampus in an Alzheimer's disease mouse model. Magn Reson Imaging 2018;50:26-37.

20. Keihaninejad S, Zhang H, Ryan NS, et al. An unbiased longitudinal analysis framework for tracking white matter changes using diffusion tensor imaging with application to Alzheimer's disease. Neuroimage 2013;72:153-63.

21. Nowrangi MA, Lyketsos CG, Leoutsakos JM, et al. Longitudinal, region-specific course of diffusion tensor imaging measures in mild cognitive impairment and Alzheimer's disease. Alzheimers Dement 2013;9:519-28.

22. Yang D, Xie Z, Stephenson D, et al. Volumetric MRI and MRS provide sensitive measures of Alzheimer's disease neuropathology in inducible Tau transgenic mice (rTg4510). Neuroimage 2011;54:2652-8.

23. Shen Z, Lei J, Li X, et al. Multifaceted assessment of the APP/PS1 mouse model for Alzheimer's disease: Applying MRS, DTI, and ASL. Brain Res 2018;1698:114-20. 
24. Drzezga A, Becker JA, Van Dijk KR, et al. Neuronal dysfunction and disconnection of cortical hubs in nondemented subjects with elevated amyloid burden. Brain 2011;134:1635-46.

25. Holland JP, Liang SH, Rotstein BH, et al. Alternative approaches for PET radiotracer development in Alzheimer's disease: imaging beyond plaque. J Labelled Comp Radiopharm 2014;57:323-31.

26. Matharu B, Spencer N, Howe F, et al. Gadoliniumcomplexed A $\beta$-binding contrast agents for MRI diagnosis of Alzheimer's Disease. Neuropeptides 2015;53:63-70.

27. Murray ME, Lowe VJ, Graff-Radford NR, et al. Clinicopathologic and 11C-Pittsburgh compound B implications of Thal amyloid phase across the Alzheimer's disease spectrum. Brain 2015;138:1370-81.

28. Fleisher AS, Chen K, Liu X, et al. Using positron emission tomography and florbetapir F18 to image cortical amyloid in patients with mild cognitive impairment or dementia due to Alzheimer disease. Arch Neurol 2011;68:1404-11.

29. Zhong Y, Karlawish J, Johnson MK, et al. The Potential Value of $\beta$-Amyloid Imaging for the Diagnosis and Management of Dementia: A Survey of Clinicians. Alzheimer Dis Assoc Disord 2017;31:27-33.

30. Ossenkoppele R, Jansen WJ, Rabinovici GD, et al. Prevalence of amyloid PET positivity in dementia syndromes: a meta-analysis. JAMA 2015;313:1939-49.

31. Jansen WJ, Ossenkoppele R, Knol DL, et al. Prevalence of cerebral amyloid pathology in persons without dementia: a meta-analysis. JAMA 2015;313:1924-38.

32. Bateman RJ, Xiong C, Benzinger TL, et al. Clinical and biomarker changes in dominantly inherited Alzheimer's disease. N Engl J Med 2012;367:795-804.

33. Spencer NG, Bridges LR, Elderfield K, et al. Quantitative evaluation of MRI and histological characteristics of the $5 \mathrm{xFAD}$ Alzheimer mouse brain. Neuroimage 2013;76:108-15.

34. Jack CR Jr, Wengenack TM, Reyes DA, et al. In vivo magnetic resonance microimaging of individual amyloid plaques in Alzheimer's transgenic mice. J Neurosci 2005;25:10041-8.

35. Boretius S, Kasper L, Tammer R, et al. MRI of cellular layers in mouse brain in vivo. Neuroimage 2009;47:1252-60.

36. Lee SP, Falangola MF, Nixon RA, et al. Visualization of beta-amyloid plaques in a transgenic mouse model of Alzheimer's disease using MR microscopy without contrast reagents. Magn Reson Med 2004;52:538-44.

37. House MJ, St Pierre TG, Kowdley KV, et al. Correlation of proton transverse relaxation rates (R2) with iron concentrations in postmortem brain tissue from alzheimer's disease patients. Magn Reson Med 2007;57:172-80.

38. van Duijn S, Bulk M, van Duinen SG, et al. Cortical Iron Reflects Severity of Alzheimer's Disease. J Alzheimers Dis 2017;60:1533-45.

39. Everett J, Céspedes E, Shelford LR, et al. Evidence of redox-active iron formation following aggregation of ferrihydrite and the Alzheimer's disease peptide $\beta$-amyloid. Inorg Chem 2014;53:2803-9.

40. Everett J, Céspedes E, Shelford LR, et al. Ferrous iron formation following the co-aggregation of ferric iron and the Alzheimer's disease peptide $\beta$-amyloid (1-42). J R Soc Interface 2014;11:20140165.

41. Dhenain M, El Tannir El Tayara N, Wu TD, et al. Characterization of in vivo MRI detectable thalamic amyloid plaques from APP/PS1 mice. Neurobiol Aging 2009;30:41-53.

42. Faber C, Zahneisen B, Tippmann F, et al. Gradient-echo and CRAZED imaging for minute detection of Alzheimer plaques in an APPV717I x ADAM10-dn mouse model. Magn Reson Med 2007;57:696-703.

43. Liu B, Moloney A, Meehan S, et al. Iron promotes the toxicity of amyloid beta peptide by impeding its ordered aggregation. J Biol Chem 2011;286:4248-56.

44. Telling ND, Everett J, Collingwood JF, et al. Iron Biochemistry is Correlated with Amyloid Plaque Morphology in an Established Mouse Model of Alzheimer's Disease. Cell Chem Biol 2017;24:12051215.e3.

45. Benveniste H, Einstein G, Kim KR, et al. Detection of neuritic plaques in Alzheimer's disease by magnetic resonance microscopy. Proc Natl Acad Sci U S A 1999;96:14079-84.

46. Vanhoutte G, Dewachter I, Borghgraef P, et al. Noninvasive in vivo MRI detection of neuritic plaques associated with iron in APPV717I transgenic mice, a model for Alzheimer's disease. Magn Reson Med 2005;53:607-13.

47. Deistung A, Schweser F, Reichenbach JR. Overview of quantitative susceptibility mapping. NMR Biomed 2017. doi: 10.1002/nbm.3569.

48. Gong NJ, Kuzminski S, Clark M, et al. Microstructural alterations of cortical and deep gray matter over a season of high school football revealed by diffusion kurtosis imaging. Neurobiol Dis 2018;119:79-87.

49. Haacke EM, Liu S, Buch S, et al. Quantitative susceptibility mapping: current status and future directions. Magn Reson Imaging 2015;33:1-25. 
50. Haacke EM, Ayaz M, Khan A, et al. Establishing a baseline phase behavior in magnetic resonance imaging to determine normal vs. abnormal iron content in the brain. J Magn Reson Imaging 2007;26:256-64.

51. Forouzannezhad P, Abbaspour A, Fang C, et al. A survey on applications and analysis methods of functional magnetic resonance imaging for Alzheimer's disease. J Neurosci Methods 2019;317:121-40.

52. Gauthier CJ, Fan AP. BOLD signal physiology: Models and applications. Neuroimage 2019;187:116-27.

53. Braak H, Alafuzoff I, Arzberger T, et al. Staging of Alzheimer disease-associated neurofibrillary pathology using paraffin sections and immunocytochemistry. Acta Neuropathol 2006;112:389-404.

54. Sperling RA, Laviolette PS, O'Keefe K, et al. Amyloid deposition is associated with impaired default network function in older persons without dementia. Neuron 2009;63:178-88.

55. Buckner RL, Sepulcre J, Talukdar T, et al. Cortical hubs revealed by intrinsic functional connectivity: mapping, assessment of stability, and relation to Alzheimer's disease. J Neurosci 2009;29:1860-73.

56. Mori S, Zhang J. Principles of diffusion tensor imaging and its applications to basic neuroscience research. Neuron 2006;51:527-39.

57. Seeley WW, Crawford RK, Zhou J, et al. Neurodegenerative diseases target large-scale human brain networks. Neuron 2009;62:42-52.

58. Raj A, Kuceyeski A, Weiner M. A network diffusion model of disease progression in dementia. Neuron 2012;73:1204-15.

59. Salek RM, Xia J, Innes A, et al. A metabolomic study of the CRND8 transgenic mouse model of Alzheimer's disease. Neurochem Int 2010;56:937-47.

60. Metastasio A, Rinaldi P, Tarducci R, et al. Conversion of MCI to dementia: Role of proton magnetic resonance spectroscopy. Neurobiol Aging 2006;27:926-32.

61. Xu W, Zhan Y, Huang W, et al. Reduction of hippocampal $\mathrm{N}$-acetyl aspartate level in aged APP(Swe)/PS1(dE9) transgenic mice is associated with degeneration of CA3 pyramidal neurons. J Neurosci Res 2010;88:3155-60.

62. Chen SQ, Cai Q, Shen YY, et al. Age-related changes in brain metabolites and cognitive function in APP/PS1 transgenic mice. Behav Brain Res 2012;235:1-6.

63. van Zijl PCM, Lam WW, Xu J, et al. Magnetization Transfer Contrast and Chemical Exchange Saturation Transfer MRI. Features and analysis of the field-dependent saturation spectrum. Neuroimage 2018;168:222-41.

64. Wang R, Wang C, Dai Z, et al. An Amyloid- Targeting Chemical Exchange Saturation Transfer Probe for In Vivo Detection of Alzheimer's Disease. ACS Chem Neurosci 2019;10:3859-67.

65. Akkus Z, Galimzianova A, Hoogi A, et al. Deep Learning for Brain MRI Segmentation: State of the Art and Future Directions. J Digit Imaging 2017;30:449-59.

66. Burton EJ, Barber R, Mukaetova-Ladinska EB, et al. Medial temporal lobe atrophy on MRI differentiates Alzheimer's disease from dementia with Lewy bodies and vascular cognitive impairment: a prospective study with pathological verification of diagnosis. Brain 2009;132:195-203.

67. Ramzan F, Khan MUG, Rehmat A, et al. A Deep Learning Approach for Automated Diagnosis and MultiClass Classification of Alzheimer's Disease Stages Using Resting-State fMRI and Residual Neural Networks. J Med Syst 2019;44:37.

68. Mossello E, Ballini E. Management of patients with Alzheimer's disease: pharmacological treatment and quality of life. Ther Adv Chronic Dis 2012;3:183-93.

69. Li M, Guan Y, Zhao A, et al. Using Multifunctional Peptide Conjugated Au Nanorods for Monitoring $\beta$-amyloid Aggregation and Chemo-Photothermal Treatment of Alzheimer's Disease. Theranostics 2017;7:2996-3006.

70. Barage SH, Sonawane KD. Amyloid cascade hypothesis: Pathogenesis and therapeutic strategies in Alzheimer's disease. Neuropeptides 2015;52:1-18.

71. Jacobsen H, Ozmen L, Caruso A, et al. Combined treatment with a BACE inhibitor and anti-A antibody gantenerumab enhances amyloid reduction in APPLondon mice. J Neurosci 2014;34:11621-30.

72. Sun D, Qiao Y, Jiang X, et al. Multiple Antigenic Peptide System Coupled with Amyloid Beta Protein Epitopes As An Immunization Approach to Treat Alzheimer's Disease. ACS Chem Neurosci 2019;10:2794-800.

73. Ricciarelli R, Fedele E. The Amyloid Cascade Hypothesis in Alzheimer's Disease: It's Time to Change Our Mind. Curr Neuropharmacol 2017;15:926-35.

Cite this article as: $\mathrm{Yu}$ B, Shan Y, Ding J. A literature review of MRI techniques used to detect amyloid-beta plaques in Alzheimer's disease patients. Ann Palliat Med 2021;10(9):1006210074. doi: 10.21037/apm-21-825 